\title{
Design and Implement of Laboratory Management System based Web
}

\author{
Zheng-Bo LI \\ School of Economic Management, Beihua University, Jilin 132013, China.
}

KEYWORD: Laboratories management; Web; Platform construction

\begin{abstract}
The laboratories are an important part in university because the quality of the facilities and their management of the laboratories would directly affect the quality of teaching and research of the institution. The functional modules have been developed, based on a functional analysis of the characteristics and actual situation of the laboratory management, using a Webbased, object-oriented and modulated designing approach, namely, the system management, the labteaching management, the lab-information management, research information management, and the file management modules, which are made into Web-based laboratory management information system that combines the $\mathrm{B} / \mathrm{S}$ and the $\mathrm{C} / \mathrm{S}$ modes. Test shows that the system meets the final proposed foals.
\end{abstract}

\section{GENERAL INSTRUCTIONS}

Lab management of various colleges and universities vary, but the scientific management, standardization perspective should follow in line with national standards laboratory construction and management, follow relevant national standards in the system implementation process, but also Universities use various management information system functional integration and data exchange. The present system therefore functions involved in the system requirements are the Ministry of Education, "Colleges Basic Labs Evaluation Criteria" and "Selected colleges laboratory work rules" as a guide to achieve the Experimental Teaching mouth often work services the purpose of scientific research, its content needs to relate to the occurrence of contact with the experimental teaching aspects, such as the staff and management of student information, establish accounts registration and management of all types of equipment and facilities, experimental teaching process dynamic information, laboratory bear management project information, laboratory rules and regulations, documents and other information. Strive to make the entire laboratory work to achieve standardized management, science, improve the efficiency of experimental teaching sector, optimize the allocation of laboratory equipment and facilities, timely feedback on the experimental teaching process problems and achieve traceability experimental teaching process for decisionmaking department provide true and reliable information on the basis of promoting laboratories, promoting experimental Teaching work, experimental teaching research work.

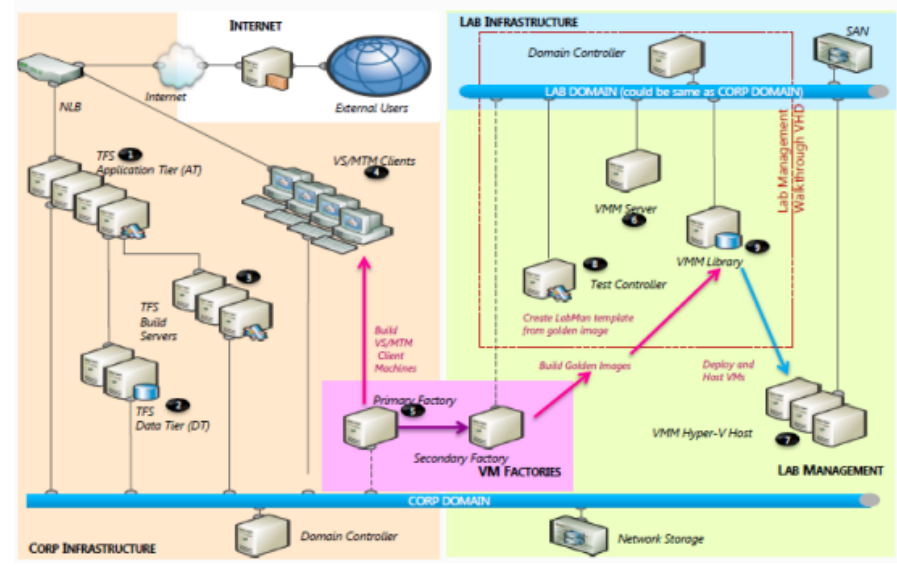

Figure 1. Configuring and Administering Lab Management

To achieve these requirements, the specific requirements of a particular author combines practical laboratory management units as well as some other universities laboratory management, exploration and development of "WEB-based laboratory management information system", the system uses B / S Mode and C / S model of combining heterogeneous model to ASP + SQL 
SERVER major technology development environment. Since the laboratory management system is a network system design, the system is a complex task based on the author's main task is to carry out the work and the overall system analysis and design, and is responsible for the design and implementation of the work of several major subsystems. mainly include:

1. According to the characteristics of laboratory management and the creation of a new research laboratory information and management system;

2. The need to create a model and divided into several modules;

3. Planning and design laboratory management database;

4. Laboratory management system design subsystems and partially achieved.

With the continuous expansion of the scale of universities, high-tech development led to laboratory testing technology, equipment and the rapid development of data applications, laboratory information network management has become an essential supporting conditions. To improve the management level and quality, protection of teaching and research carried out smoothly play a positive role, a certain application and application value.

1. Standardized laboratory management and improve office efficiency and meet the needs of management system. For a long time to communicate with common laboratory management laboratory sluggish, inconvenient exchanges and other issues, many public laboratories, specialized laboratories, experimental research base on the lab and equipment management still remain in a single computer and artificial combination of management stage, the main form of management dominated by administrative means, this management model because there is no network, only increasing the workload of lab personnel, we can not make full use of laboratory resources, resulting in a relative waste. In addition, as the school's development in recent years, space and distance has become very prominent in laboratory management and pressing problem.

2. Meet the needs of laboratory modernization. In the past the majority of equipment manufacturers test machine control software is a stand-alone version of the software, do not have the network information transmission function, the network can not meet the needs of laboratory construction. Now many experiments and research base and production center jointly developed a network version control software for network provide the necessary conditions. When the laboratory has advanced laboratory equipment, how to establish a reliable information channel in order to achieve the lowest possible effective messaging has become the main topic of laboratory construction. As an important symbol of modern information, computer network technology for the efficient transmission of information to provide the basic conditions.

3. The third is the need to adapt to the experimental teaching reform. Application of information technology, which greatly promoted the reform of college, and as a practical experiment teaching, due to the experimental space and equipment layout, student and instructor experiment dispersed in one or even several large environment, experiment centralized monitoring difficult process, experimental data scattered in different experimental apparatus, difficulty in concentrating management and analysis, is not conducive to improve the effectiveness of experimental teaching. Meanwhile, simulation, virtual experiments, remote experiment teaching has become one of the development direction of modern experimental teaching reform, regardless of which of the experimental teaching reform are inseparable from the computer, computer network can not do without, we must use the network information transmission.

4. The fourth is to give full play the role of a comprehensive laboratory. Set up a network, information-based laboratory information network management platform, which is designed not only to achieve the network share information, the paperless office, reducing resource management costs, reduce social fixed assets investment, make money investment to maximize efficiency, providing the technical basis for significantly improved laboratory and laboratory management efficiency and enhance the overall level of equipment management, more important is to help people change the traditional concept of the beginning of a new way of working, so that more and more people are enjoying new ways to bring convenience, efficiency and happiness.

\section{SYSTEM DEVELOPMENT PLATFORM RELATED TECHNOLOGIES}

Laboratory Management System built environment and the main technical solutions adopted, the system based on B / S and C / S mixed-mode application development technologies at work IInternet environment. Web technology and interactive technology Web database application 
system is the key to achieve, on the technical versatility, ease of development, and load balance to consider, and to the security of the Web environment and database systems were discussed.

With the development of network technology and the Internet work, work-based Internet technology has almost become a unified model for all the computer network and the de facto technology standard. 1990s, the traditional network client / server architecture to the development of technology-based Internet network environment, the mid-1990s, Internet web applications have been identified by the international community as a new message mode - browser / server (B / S mode, WEB and the World Wide Web is the world's largest information system all users have a unified client and browser .Web has become WAN and intranet to access and publish information. important means now develop most applications are Web-based interactive applications, so the combination of Web technology and database technology more closely, more and more traditional database information management system (M Engineering S) are porting to the web, so the use of web database technology to establish a secure laboratory information management system is an important research topic.

C/S (Client/Server, Client/Server) structure, which is well-known client and server architecture, is very mature application architecture is widely used in various information management systems. It is two-tier architecture; it is divided into client and server-side two parts, both of which are connected by a local area network. The front-end client applications mainly display interface, and user interaction, and a background server process is mainly responsible for data processing, postprocessing the results returned to the user. In this configuration, the server hardware must have sufficient processing capacity, so as to meet the requirements of customers.

\section{WEB DATABASE TECHNOLOGY OVERVIEW}

Web database development dynamic pages generally refer to the use of the database. Simply put, is to use the browser as a data input interface, user input required, the browser will return this data to the Web server, Web server and then the data is processed, for example, to modify the data in the database or in the database data query, the results of the final site will be executed back to the browser, the browser displayed to the user.

Like the common database, Web database is stored data system, in fact, Web database system used and usually we used the database system is the same. The more popular Web database system has the SQL Server, Oracle, Access and FoxPro, one of the most commonly used is Access and SQL Server.

Web developers usually use the browser / server (B / S framework of this architecture the client uses the browser provides the user interface, unified style, low client requirements;. The server uses Web server that provides Web-based dynamic pages, centralized management, develop flexible. Typical applications often need to access the database, to reduce the burden on the server, you can Web server and database server separated, resulting in a three-layer structure in which the client uses the browser sends a request to the Web server, Web server connect to the database server, database server access to data, the final form page returned to the client this structure has the following advantages:

ince the relational database at the beginning is not directed against workers Internet design, so connect to the database via the Web, usually requires the use of an intermediate interface. Commonly used include ODBC (Open Database Connectivity, Open Database Connectivity), JDBC (Java Database Connectivity, Java Database Connectivity) and OLE DB and so on. Connection interface with different Web development platform used is also different; write serverside applications have a very important role in the development of Web database system, the effect of the application of a direct impact on the quality of customer service and service. Such applications must have the following functions:

1. To establish contact with the back-end database and release

2. Can send data to back-end database operating instruction

3. The request can be sent to customers respond

4. Can handle returned from the database information

5. Can handle data from customer feedback 


\section{ASP AND SQL DATABASE CONNECTION}

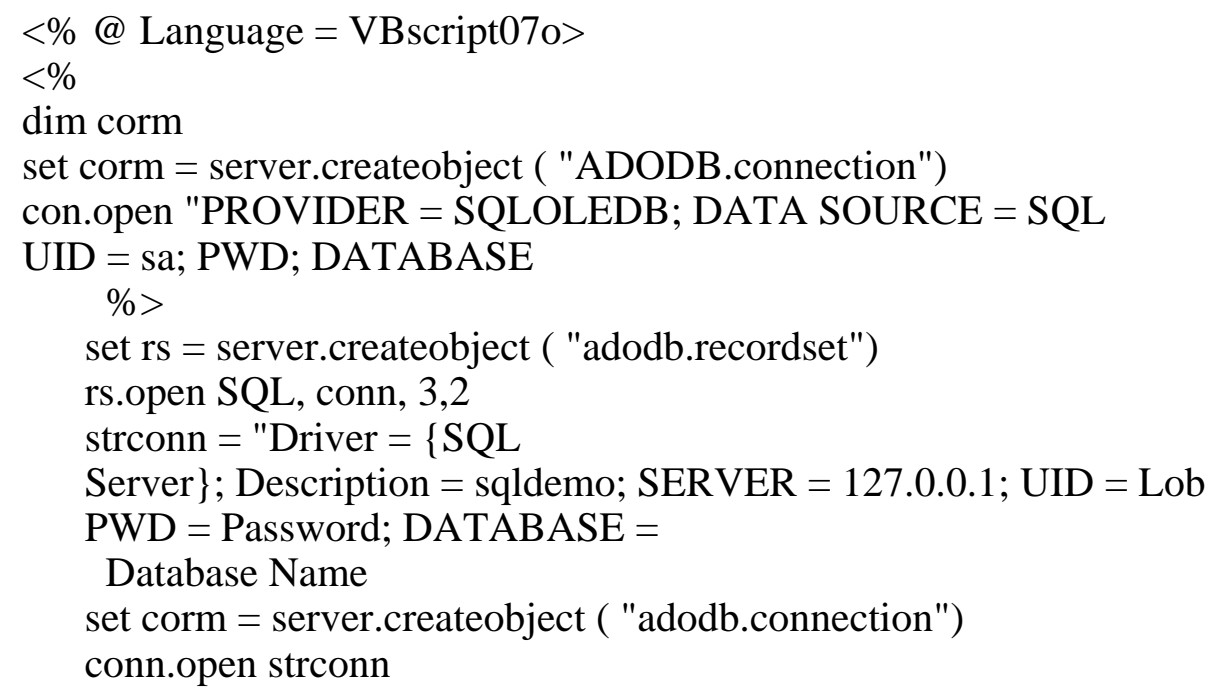

\section{IMPLEMENTATION OF WEB-BASED LAB MANAGEMENT SYSTEM}

A good, stable environment enables development of the entire system efficiently and quickly completed, while the development of quality systems can be well protected. The development environment was constructed as follows.

Hardware environment

1 , the server

The main server configuration:

Processor: Workers ntel pentium 4

Memory: 512M and above

Hard disk: 80G more

Graphics: SVGA graphics adapter

LAN: 100Mbps

2 , the client

The client side of the main configurations:

Processor: Workers ntel pentium 4

Memory: 512M and above

Hard disk: 40G more

Graphics: SVGA graphics adapter

LAN: 100Mbps

Software Environment

1 , the server

The main server configuration:

Operating System: Windows Server 2000/Windows Server 2003, support WEB SERVER ASP script.

Network protocols: TCP / worker P

WEB SERVER: Workers S5 X or IIS6 X; support the asp web server

Database: Microsoft SQL Server 2000

2, the client

The client side of the main requirements:

Operating System: windows 2000 / windows xp

Network protocols: TCP / worker P

Browser: Internet Explorer 5 and above (support frame)

\section{FUNCTION MODULE TO REALIZE}

Here only for system information management module functions will be described:

1 , user login 
The main program code is as follows

private void ImageButtonl-click (object sender,

System.Web.Ul.ImageClickEventArgse)

\{

String se1Sq1;

Sql Conneetion Sq1Con = new Sql Conneetion (Sq1Dsn);

//Response.Write(Sq1Dsn);

Sql Con.open ():

selsql $=$ 'SELECT * FROMLabUserWHERE name $=$," + TextBox3.Text

Password = " '+ TextBox4.Text +" "':

SqlCommandcmd = newSqlConunand (se1Sq1, Sq1Con);

. SqlDataReadersdr $=$ cmd Execute Reader ():

if (sdr.Read ())

\{

Session [ "name"] = TextBox3.Text;

Label7.Text = "Login successful";

Label6.Text = "online now Administrator:" + TextBox3.Text;

//Response.Redireet("jiaoshixinxi.aspx ");

//Response.Write("OK ");

e1Se

Session ["sysbh"] = "';

Labe 16.Text = "';

Label7.Text = ', user name or password is incorrect, please re-enter! ".;

TextBox3.Text = "';

TextBox4.Text = "';

\}

sdr.Close ();

\}

\section{SYSTEM TEST}

Purpose of software testing is to find out as much as possible with the least cost potential software errors and defects; it has the practicality and stability. Tests, mainly from the following aspects of the system were tested:

1. Test form

As a database system, the operation is the most basic form is the most frequent operation, whether to submit the required information accurately is the key to whether the system can achieve the desired objectives. By storing the data of the respective units, extraction, query tested, indicating that the system is operating normally.

2. Link test

Links are a key feature of Web application system, which guarantees the system data switch between pages. Link test first tests whether all links by indicating that indeed link to the links page; Secondly, test the contents of the linked page display is wrong; link test must be done in the integration testing phase, that is to say, the entire Web application system after the completion of the development of all the pages link to test.

3. System testing

The software system is a whole to be tested. After the system is substantially complete, take SIMULATION working environment was simulated test work, and according to the design characteristics of mouth is often the work of the test cases, the main function of the basic system can be realized. The following query device and an example to illustrate.

\section{REFERENCES}

Ren Yi, Liu Xiaohai, Liao listen based on C/S and B/S hybrid architecture with examples of analysis Computer Engineering and Applications 2001 (16): 159-160 
Pengyue Ping Design Laboratory Information Management System CS and BS binding mode of the microprocessor-based electrical and computer science, 2006,23 (8): 187-189

$\mathrm{Xu}$ Scots, had feathers, a Pender Software Engineering Theory and Practice of Beijing: Higher Education Press, 2004: 24

Zhao Qing double, smell Spark, Liang Guohua Development and Application of Experimental Technology and Management Laboratory Information Management Systems, 2000, 17 (6): 3032

Pengrui Dong, Maoling Tao, Ju Young Design and implementation of database management laboratory experimental techniques and management 200421 (2): 172-177

Tang Chenghua, Chen degrees, Chen new management information system research and multi-user rights management Computer Application Research, 2004, (3): 217-219 